\title{
Phase Equilibrium in the Binary System Polyisoprene + Dioxane
}

\author{
Noriko TaKano, Yoshiyuki EINAGA, and Hiroshi FuJITA \\ Department of Macromolecular Science, Osaka University, \\ Toyonaka, Osaka 560, Japan
}

(Received May 10, 1985)

\begin{abstract}
The Flory-Huggins interaction parameter $\chi$ was formulated as a function of concentration, temperature, and chain length $(P)$ on the basis of light scattering data for two monodisperse polyisoprene samples in dioxane in the region encompassing the $\theta$ temperature. It was found that when extrapolated to the immiscibility region, the resulting $\chi$ predicts binodals, spinodals, and critical points which agree almost perfectly with observed results. Another important finding is that $\chi$ depends distinctly on $P$ even at high concentrations where chain overlapping takes place.
\end{abstract}

KEY WORDS Phase Equilibrium / Flory-Huggins Interaction Parameter / Cloud Point Curve / Binodal / Spinodal / Critical Point / Theta Temperature / Light Scattering / Polyisoprene / Dioxane /

The Flory-Huggins interaction parameter $\chi$ still occupies the central seat in polymer thermodynamics. ${ }^{1}$ Many expressions have been proposed for it and tested against experiment. $^{2-6}$ However, few of them have achieved more than a limited success in quantitative prediction of observed phase relationships, even for quasibinary polymer solutions.

Recently, we have launched a series of investigations aiming at the formulation of $\chi$ for such solutions on the basis of experimental information mainly from light scattering measurements. ${ }^{7,8}$ Our spirit is essentially akin to that of Scholte, ${ }^{9,10}$ which led to a series of important contributions, but we primarily focus on the behavior of solutions below the $\theta$ temperature.

Thus, in a recent study $^{7}$ dealing with a binary system consisting of monodisperse (actually, very narrow-distribution) polystyrene (PS) and cyclohexane ( $\mathrm{CH})$, we formulated an empirical expression for the parameter $Z$, which is related to $\chi$ by

$$
Z=\chi+(1 / 2) \phi(\partial \chi / \partial \phi)_{\mathrm{T}}
$$

as a function of polymer volume fraction $\phi$, absolute temperature $T$, and polymer relative chain length $P$ from $\theta$ down to the cloud point. It reads

$$
\begin{aligned}
Z(T, \phi ; P)= & \chi_{\text {conc }}^{0}(T ; P)+(1 / 2) \phi \\
& +A(P) \phi^{4} /\left[1+B(T ; P) \phi^{2}\right] \\
& +\left[\chi_{\mathrm{dil}}^{0}(\mathrm{~T})-\chi_{\mathrm{conc}}^{0}(T ; P)\right] R(\phi ; P)
\end{aligned}
$$

with $\chi_{\text {dil }}^{0}, \chi_{\text {conc }}^{0}, A, B$, and $R$ being determined from light scattering data. It was shown that $\chi$ calculated from eq 1 and 2 gives almost quantitative predictions of some typical data for binodals, spinodals, and critical points, with no parameter adjustment.

Having been encouraged by this success, we undertook a similar study, this time choosing polyisoprene (PIP) + dioxane as a test system, to see whether eq 2 is applicable for binary systems other than PS $+\mathrm{CH}$. The results obtained are reported in the present paper. 


\section{EXPERIMENTAL}

\section{Polyisoprene Samples}

Dr. M. Fukuda of Toyo Soda Co. kindly made two laboratory PIP samples P-5 and P13 disposable for our investigation. These samples contained $1 \mathrm{wt} \%$ 2,6-di- $t$-butyl- $p$ cresol as an antioxidant and were about $80 \%$ in cis content. When measured by light scattering in cyclohexane at $25^{\circ} \mathrm{C}$, the weightaverage molecular weight $M_{w}$ was $5.33 \times 10^{4}$ for sample P-5 and $13.3 \times 10^{4}$ for sample P13. The polydispersity index $M_{w} / M_{n}\left(M_{n}\right.$ is the number-average molecular weight) estimated by gel permeation chromatography was less than 1.08 for both samples. Therefore, all the experimental results obtained in this work were analyzed assuming that the two samples were monodisperse in molecular weight.

\section{Preparation of Solutions}

A given PIP sample was dried under a reduced pressure for more than four weeks. Part of it to be used for light scattering measurement was made dust free by filtering its dilute dioxane solution with a Millipore film and then by freeze drying. The solvent dioxane was carefully distilled after having been refluxed over sodium for more than one week. To avoid the contamination with moisture, test solutions, for either light scattering or phase separation experiment, were prepared by mixing known amounts of the polymer and solvent in a dry bag filled with dry nitrogen.

The volume fraction $\phi$ of the polymer in a given solution was computed by use of the equation

$$
\phi=\left[1+\left(v_{0} / v_{\mathrm{p}}\right)\left(w^{-1}-1\right)\right]^{-1}
$$

where $w$ is the weight fraction of the polymer, and $v_{0}$ and $v_{\mathrm{p}}$ the specific volumes of the solvent and polymer in the pure state, respectively. The values of 1.0280 and $1.0951 \mathrm{~cm}^{3} \mathrm{~g}^{-1},{ }^{11}$ which refer to $25^{\circ} \mathrm{C}$, were used for $v_{0}$ and $v_{\mathrm{p}}$, respectively, regardless of the temperature of the solution studied. Note that $\phi$ defined by eq 3 is not the volume fraction in the usual sense.

\section{Light Scattering Measurements}

Intensities of light scattered from PIP + dioxane solutions were measured at scattering angles from 30 to $150^{\circ}$. The range of $\phi$ investigated was from 0.027 to 0.22 for sample P-5 and from 0.018 to 0.21 for sample P-13. The measuring temperature was varied at appropriate intervals from 40 to $21^{\circ} \mathrm{C}$ for the former and from 40 to $26^{\circ} \mathrm{C}$ for the latter. A Fica 50 light scattering photometer was used with polarized incident light of $546 \mathrm{~nm}$ wavelength. The data were analyzed by using the relation $^{7}$

$$
K / \Delta R_{0}=-[1 /(R T \phi)]\left(\partial \Delta \mu_{0} / \partial \phi\right)_{\mathrm{T}}
$$

where

$$
K \equiv\left(4 \pi^{2} n^{2} / N_{\mathrm{A}} \lambda_{0}{ }^{4}\right)(\partial n / \partial \phi)_{\mathrm{T}}{ }^{2} V_{0}
$$

Here $\Delta R_{0}$ is the excess Rayleigh ratio at zero scattering angle, $\Delta \mu_{0}$ the excess chemical potential of the solvent, $R$ the gas constant, $n$ the refractive index of the solution, $N_{\mathrm{A}}$ the Avogadro constant, $\lambda_{0}$ the wavelength of incident light in vacuum, and $V_{0}$ the molar volume of the solvent.

Values of $n$ for PIP + dioxane at 21, 25, 30, and $40^{\circ} \mathrm{C}$ were measured by a Bausch-Lomb precision refractometer in the range of $w$ from 0 to 0.24 . The results were used to evaluate the $w$-basis refractive index increment $\partial n / \partial w$ as a function of $w$ and $T$, and it was found that $\partial n / \partial w$ was virtually independent of $w$ and varied linearly with $T$ according to

$$
\partial n / \partial w=(660.5+1.33 T) \times 10^{-4}
$$

With eq $3, \partial n / \partial w$ was converted to $\partial n / \partial \phi$ for the calculation of $K$.

\section{Determination of Cloud Points}

Cloud point temperatures for a series of PIP + dioxane solutions were measured in a double thermostated water bath by monitoring 
the intensity of He-Ne laser light transmitted through the solutions. The accuracy was $\pm 0.01^{\circ} \mathrm{C}$. The procedure used was the same as that described elsewhere. ${ }^{12}$

\section{RESULTS AND DISCUSSION}

\section{Chemical potential}

Plots of $K / \Delta R_{\theta}\left(\Delta R_{\theta}\right.$ is the excess Rayleigh ratio at scattering angle $\theta$ ) vs. $\sin ^{2} \theta / 2$ at each fixed $\phi$ were fitted closely by a straight line and accurately extrapolated to $\theta=0$. The values of $-(1 / R T)\left(\partial \Delta \mu_{0} / \partial \phi\right)_{\mathrm{T}}$ obtained by substituting the resulting $K / \Delta R_{0}$ values into eq 4 are plotted against $\phi$ in Figure 1 for sample P-5 and in Figure 2 for sample $\mathrm{P}-13$. At temperatures above $37^{\circ} \mathrm{C},-(1 / R T)\left(\partial \Delta \mu_{0} / \partial \phi\right)_{\mathrm{T}}$ increases monotonically with increasing $\phi$, while at temperatures below $32.5^{\circ} \mathrm{C}$, it initially decreases, passes through a minimum, and then monotonically increases with increasing $\phi$. The initial slope of each of the indicated curves gives $2\left(V_{0} / v_{\mathrm{p}}{ }^{2}\right) A_{2}$, where $A_{2}$ is the second virial coefficient of the system. In Figures 1 and 2, this slope at $34^{\circ} \mathrm{C}$ is virtually zero, leading to the conclusion that the $\theta$ temperature for the system PIP + dioxane is $34 \pm 0.5^{\circ} \mathrm{C}$.

The common ordinate intercept of the smooth curves at different $T$ in either Figure 1 or 2 agrees well with $P^{-1}$ calculated by inserting the light-scattering $M_{w}$ value determined in cyclohexane into the relation

$$
P=\left(v_{\mathrm{p}} / V_{0}\right) M_{w}
$$

This finding indicates that the test dioxane solutions of PIP were free from aggregation down to temperatures close to the cloud point. The $P$ value obtained from the intercept was 681 for sample P-5 and 1700 for sample P-13. These $P$ values are used throughout the subsequent data analysis and computations.

\section{Formulation of $Z$}

For strictly binary solutions the parameter $Z$ defined by eq 1 can be related to experimentally measurable quantities $\phi, P$, and $\Delta R_{0} / K$ by*1

$\mathrm{Z}=(1 / 2)\left[1 /(1-\phi)+1 /(P \phi)-K / \Delta R_{0}\right]$

Figures 3 and 4 show $Z$ vs. $\phi$ plots for samples P-5 and P-13, respectively, obtained by substituting the data of Figures 1 and 2 into eq 8 (note eq 4 for the term $K / \Delta R_{0}$ ). Though there exist some subtle differences, the overall features of these graphs are similar to those for the system PS $+\mathrm{CH}$ below the $\theta$ temperature. $^{7}$

It is convenient for formulating $Z$ to analyze the experimental data in terms of $Y$ defined by

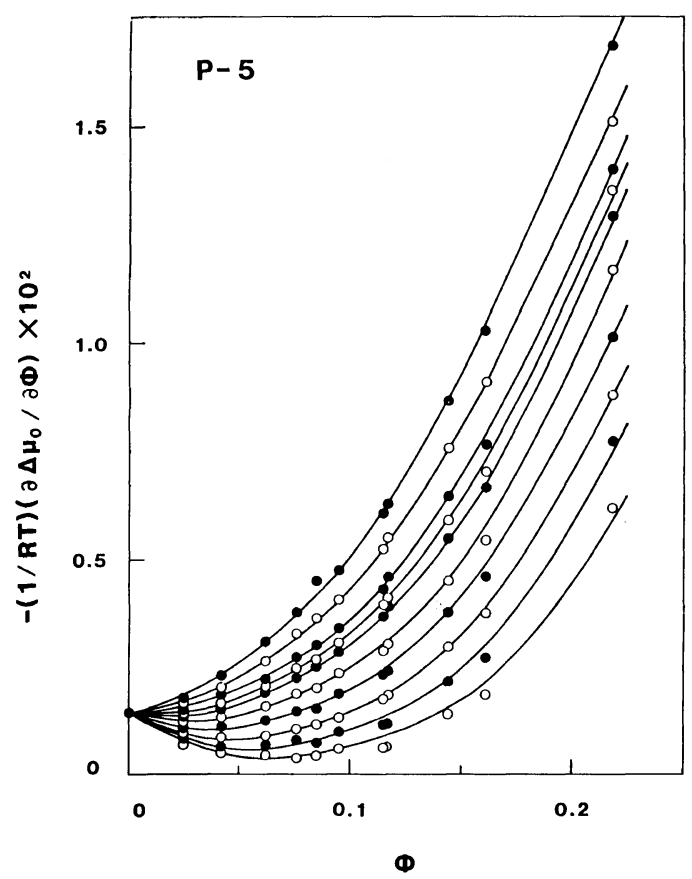

Figure 1. Plots of $-(1 / R T)\left(\partial \Delta \mu_{0} / \partial \phi\right) v s . \phi$ for sample $\mathrm{P}-5$ in dioxane. The temperature is $40,37,34,32.5,31.5$, $29,27,25,23$, and $21^{\circ} \mathrm{C}$ from top to bottom.

*1 For quasibinary solutions, eq 8 does not hold even if $P$ is replaced by $P_{w}$, the weight-average chain length of the polymer mixture (this replacement prevents $Z$ from diverging to infinity at $\phi=0$ ). Thus, in our recent work, ${ }^{13}$ we have redefined $Z$ by eq 8 with $P$ replaced by $P_{w}$. It can be shown that $Z$ so defined is no longer related to $\chi$ by eq 1 except for strictly binary solutions, but becomes a complex function of $\chi, \partial \chi / \partial \phi_{i}$, and $\left\{\phi_{i}\right\}$, where $\left\{\phi_{i}\right\}$ denotes the set of composition variables. 


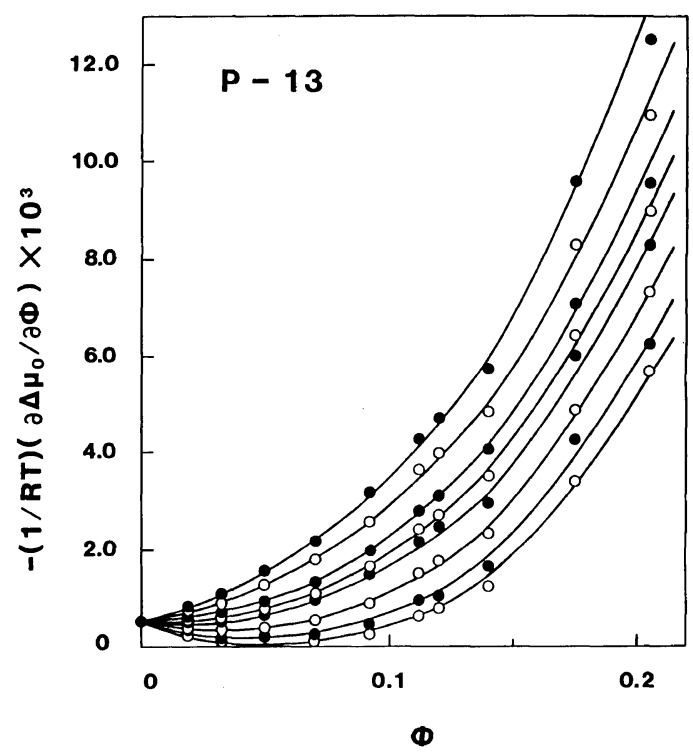

Figure 2. Plots of $-(1 / R T)\left(\partial \Delta \mu_{0} / \partial \phi\right) v s . \phi$ for sample $\mathrm{P}-13$ in dioxane. The temperature is $40,37,34,32.5,31.5$, 29,27 , and $26^{\circ} \mathrm{C}$ from top to bottom.

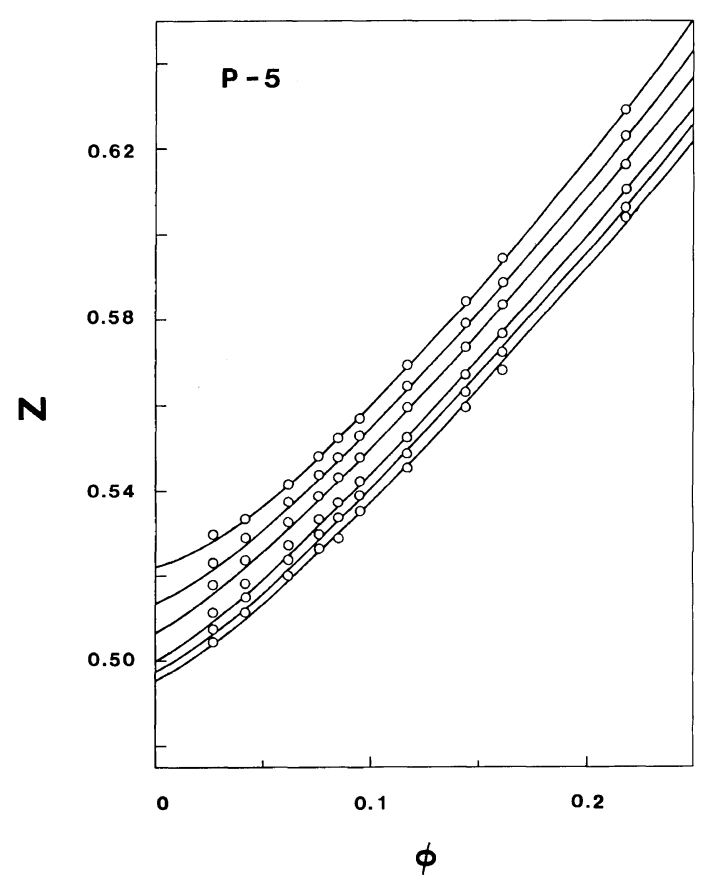

Figure 3. Concentration dependence of $Z$ for sample P-5 dioxane. The temperature is $21,25,29,34,37$, and $40^{\circ} \mathrm{C}$ from top to bottom. Solid curves indicate the calculated values.

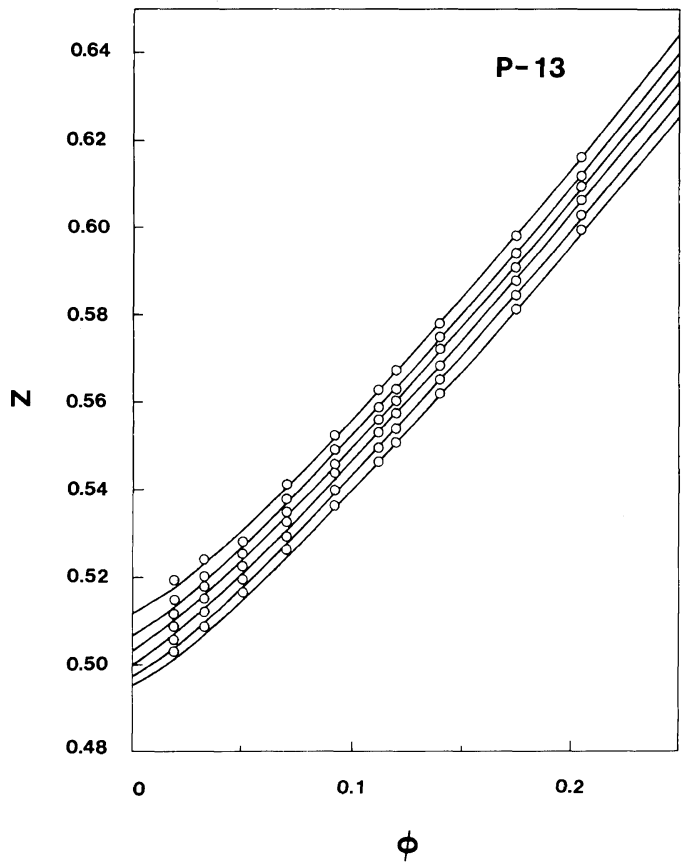

Figure 4. Concentration dependence of $Z$ for sample $\mathrm{P}-13$ in dioxane. The temperature is $26,29,31.5,34,37$, and $40^{\circ} \mathrm{C}$ from top to bottom. Solid curves indicate the calculated values.

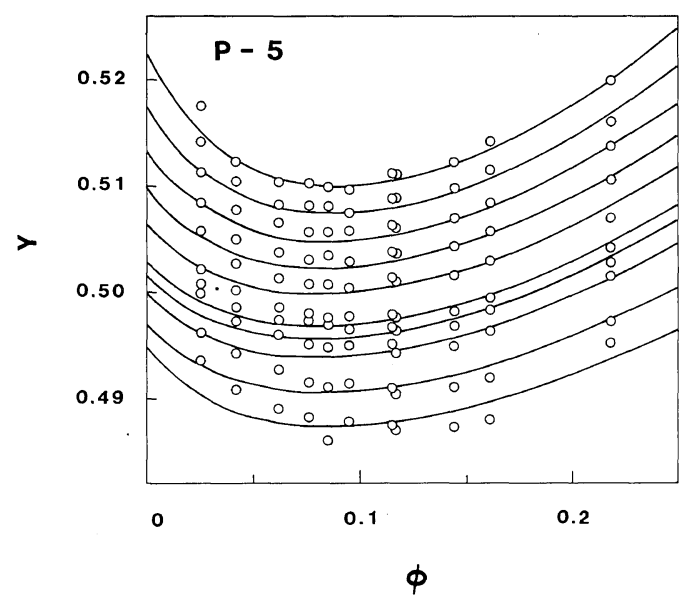

Figure 5. Plots of $Y v s$. $\phi$ for sample P-5. The temperature is $21,23,25,27,29,31.5,32.5,34,37$, and $40^{\circ} \mathrm{C}$ from top to bottom. Solid lines indicate the calculated values.

$$
Y=Z-(1 / 2) \phi
$$

Figures 5 and 6 depict $Y v s . \phi$ plots for the two samples. It can be seen that these plots for 


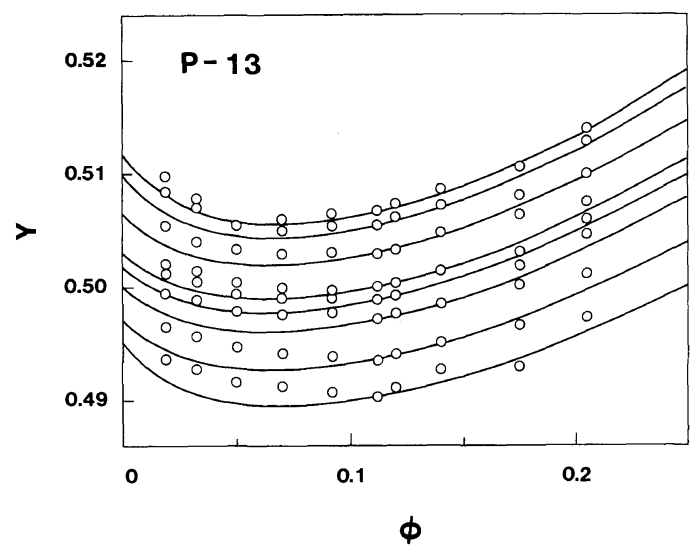

Figure 6. Plots of $Y$ vs. $\phi$ for sample P-13. The temperature is $26,27,29,31.5,32.5,34,37$, and $40^{\circ} \mathrm{C}$ from top to bottom. Solid lines indicate the calculated values.

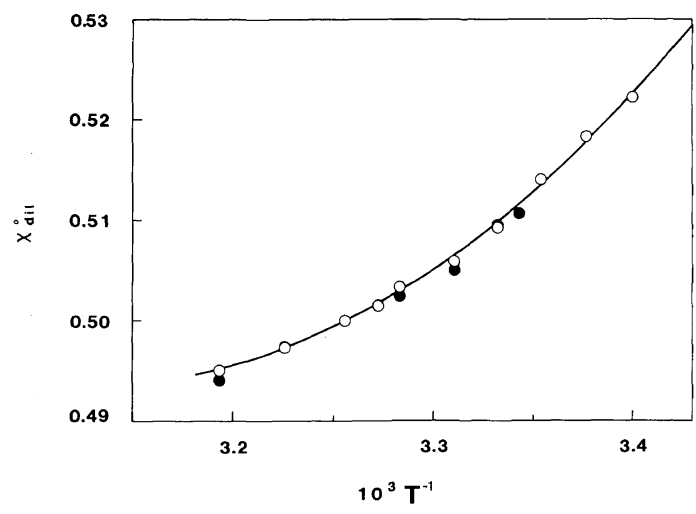

Figure 7. Parameter $\chi_{\text {dil }}^{\circ}$ plotted against $T^{-1}$ for samples P-5 (unfilled circles) and P-13 (filled circles). The solid line shows the values calculated by eq 10 .

either sample at different temperatures follow nearly parallel curves which pass shallow minima at about 0.1 of $\phi$.

In eq $2, R$ is a function approaching unity as $\phi \rightarrow 0$, so that $Z$ at $\phi=0$ gives $\chi_{\text {dil }}^{0}$. Figure 7 shows $\chi_{\text {dil }}^{0}$ extrapolated from Figures 3 and 4 as a function of $1 / T$. Interestingly, the data points for the two samples fall approximately on a single curve, which can be represented by

$$
\begin{aligned}
\chi_{\mathrm{dil}}^{0}(T)= & 0.5+0.33(\theta / T-1) \\
& +4.1(\theta / T-1)^{2}
\end{aligned}
$$

Since $A_{2}$ is related to $\chi_{\text {dil }}^{0}$ by

$$
A_{2}=\left(V_{0} / v_{\mathrm{p}}^{2}\right)\left(1 / 2-\chi_{\mathrm{dil}}^{0}\right)
$$

this finding implies that $A_{2}$ for the PIP + dioxane system below and slightly above $\theta$ is essentially independent of molecular weight. Recently, the same fact was found by Tong et $a l .{ }^{14}$ for the PS $+\mathrm{CH}$ system below $\theta$. No available theories of polymer solutions are capable of explaining these interesting findings.

As in our previous paper ${ }^{7}$ it is assumed that the behavior of $Y$ for $\phi$ above the minimum point is represented by the sum of the first and third terms of eq 2. In other words, the last term of eq 2 , which is the strategy for formulating the dilute solution behavior of $Z$, is assumed to vanish in this concentration region. By a trial-and-error procedure the sum of the first and third terms of eq 2 was fitted to the data points for $\phi>0.1$ in Figures 5 and 6, and the following empirical expressions were established for the parameters $\chi_{\text {conc }}^{0}, A$, and $B$ :

$$
\begin{gathered}
\chi_{\text {conc }}^{0}(T ; P)=0.5-0.19 P^{-1 / 2}+0.35(\theta / T-1) \\
A(P)=2 P^{1 / 3} \\
B(T ; P)=8.73 P^{1 / 3}-600(\theta / T-1)
\end{gathered}
$$

In eq 12,13 , and 14 , the exponents to $P$ were chosen to be the same as those in the corresponding expressions for the $\mathrm{PS}+\mathrm{CH}$ system, since the present data, being limited to only two $P$ values, did not allow their unique determination. Figure 8 illustrates that the $\chi_{\text {conc }}^{0}$ values determined by curve fitting can be represented well by eq 12 .

A very important conclusion from eq $12-$ 14 is that $Z$ at high concentrations $(\phi>0.1)$ depends significantly on molecular weight. Our recent study ${ }^{7}$ and an independent study of Nies et al. ${ }^{15}$ on the system $\mathrm{PS}+\mathrm{CH}$ have documented the finding of the same fact. Thus, it seems necessary that the prevailing notion that the thermodynamic behavior of polymer solutions at high concentration is not affected by molecular weight be altered at least in the 


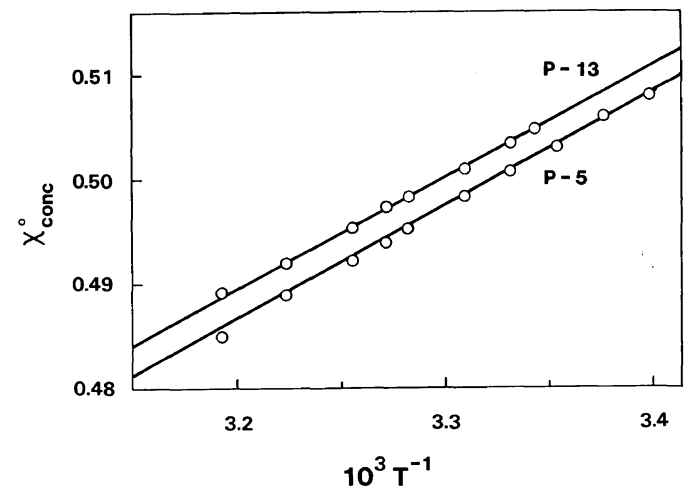

Figure 8. Parameter $\chi_{\text {conc }}^{\circ}$ plotted against $T^{-1}$. Solid lines represent the values calculated by eq 12 .

temperature region below $\theta$ or presumably in poor solvents.

The function $R$ in eq 2 was determined by plotting $\left(Y-Y_{\text {conc }}\right) /\left(\chi_{\text {dil }}^{0}-\chi_{\text {conc }}^{0}\right)$ against $\phi$, where $Y_{\text {conc }}$ denotes the sum of the first and third terms in eq 2, i.e., the concentrated solution part of $Y$, and can be evaluated with the aid of eq 12-14. It was found that the resulting plots for either sample at different temperatures are fitted approximately by a single exponential function and that these functions for the two samples are lumped by

$$
R=\exp (-K \phi)
$$

with

$$
K=3.3 P^{1 / 3}
$$

With all the parameters in eq 2 evaluated as above, we are able to compute $Z$ and $Y$ as functions of $T, \phi$, and $P$. The solid lines in Figures 3-6 show the results of these computations. Their fit to the plotted data points is only moderate at low concentrations but quite good at high concentrations.

\section{Expression for $\chi$}

Integration of eq 1 , with the requirement that $\chi$ remains finite at $\phi=0$, gives

$$
\chi=\left(2 / \phi^{2}\right) \int_{0}^{\phi} Z \phi \mathrm{d} \phi
$$

which, on substituting eq 2 with $R$ given by eq

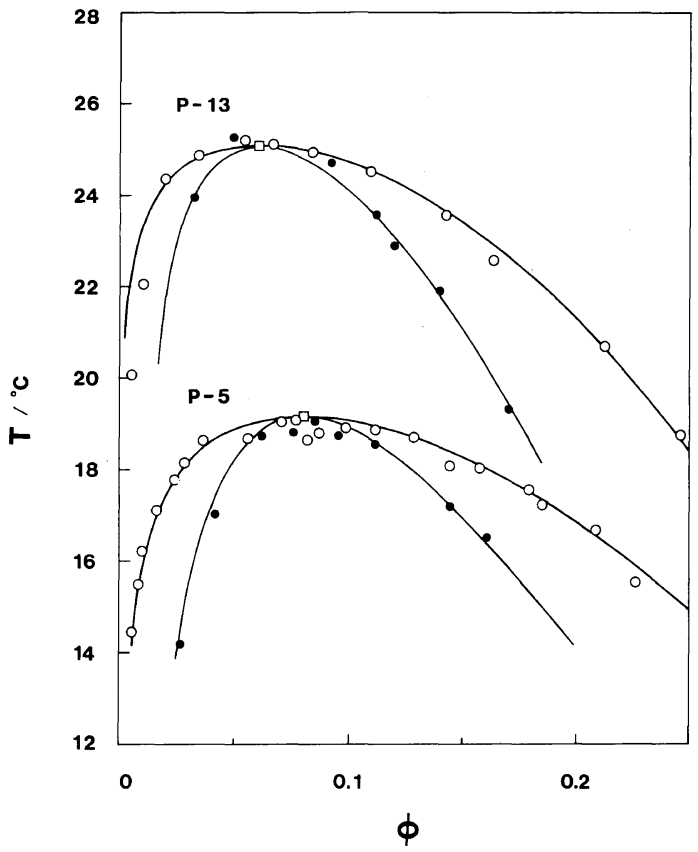

Figure 9. Cloud point curves (Binodals) (unfilled circles) and Spinodals (filled circles) for PIP samples P-5 and $\mathrm{P}-13$ in dioxane. Solid lines represent the predictions from the present theory.

15 , yields

$$
\begin{aligned}
\chi(T, \phi ; P)= & \chi_{\text {conc }}^{0}(T ; P)+(1 / 3) \phi \\
& +[A(P) / B(T ; P)]\left\{\phi^{2} / 2\right. \\
& \left.-[1 / B(T ; P)] \ln \left[1+B(T ; P) \phi^{2}\right]\right\} \\
& +\left[\chi_{\mathrm{dil}}^{0}(T)-\chi_{\text {conc }}^{0}(T ; P)\right] Q(\phi ; P)
\end{aligned}
$$

where

$Q(\phi ; P)=\left[2 /(K \phi)^{2}\right][1-(1+K \phi) \exp (-K \phi)]$

and $\chi_{\text {dil }}^{0}, \chi_{\text {conc }}^{0}, A, B$, and $K$ are given by eq 10 , $12,13,14$, and 16 , respectively.

\section{Binodals (Cloud Point Curves) and Spinodals}

Once the parameter $\chi$ is established as a function of $T, \phi$, and $P$, it is possible to calculate by familiar procedures ${ }^{1}$ any phase relationships such as binodal, spinodal, and critical point. The solid lines in Figure 9 
illustrate the binodals and spinodals for samples P-5 and P-13, calculated with $\chi$ given by eq 18 . It can be seen that they fit very closely the experimental data plotted as unfilled (cloud points) and filled (spinodal points) circles. Here, the spinodal data were obtained from Figures 1 and 2 by extrapolating the temperatures at which $\partial \Delta \mu_{0} / \partial \phi$ at given $\phi$ vanishes, i.e., $\Delta R_{0}$ diverges to infinity.

The agreement between predicted and observed results as seen in Figure 9 is distinctly better than that obtained in our previous work $^{7}$ on the PS $+\mathrm{CH}$ system. Anyway, we note that the success owes much to the fact that our formulation of $Z$ and hence $\chi$ has taken the $P$ dependence of the concentrated solution behavior properly into account.

\section{Critical Points}

The solid lines in Figures 10 and 11 show the calculated $\phi_{\mathrm{c}}$ and $T_{\mathrm{c}}^{-1}$ plotted against $P^{-1 / 3}$ and $P^{-1 / 2}$, respectively, where $\phi_{\mathrm{c}}$ and $T_{\mathrm{c}}$ denote the critical concentration and temperature. The data point on the ordinate axis in Figure 11 was obtained as the inverse $\theta$ temperature $\left(\theta=34^{\circ} \mathrm{C}\right)$, and the other data points in these graphs were determined from the peaks of the smooth curves fitting the observed cloud points shown in Figure 9. An almost complete agreement is seen between the calculated and observed values for both $\phi_{\mathrm{c}}$ and $T_{\mathrm{c}}$, as was the case in our previous work ${ }^{7}$ on the system $\mathrm{PS}+\mathrm{CH}$.

The line in Figure 11 exhibits a weak upward curvature in the region of $10^{2} P^{-1 / 2}<2$. This is contrasted to the corresponding line for the system PS $+\mathrm{CH}$, which exhibits an opposite curvature, though very weak, in the same region of $P^{-1 / 2}$. In either case, some significant error would be committed if the linear part of the $T_{\mathrm{c}}^{-1}$ vs. $P^{-1 / 2}$ plot at high $P^{-1 / 2}$ were extrapolated to $P^{-1 / 2}=0$ and the intercept were used to determine $\theta$. This gives a warning to the conventional method for estimating $\theta$ from critical temperature data. As is well known, ${ }^{16}$ a linear relation holds between $T_{\mathrm{c}}{ }^{-1}$

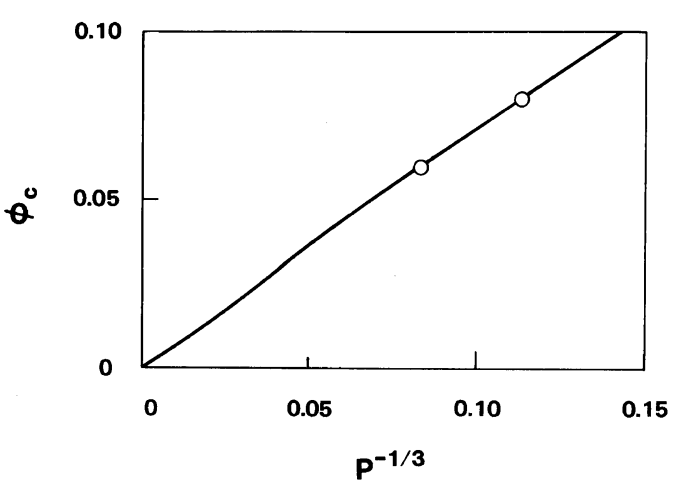

Figure 10. Critical concentration $\phi_{\text {c }}$. plotted against $P^{-1 / 3}$ for PIP in dioxane. The solid line shows the theoretical prediction.

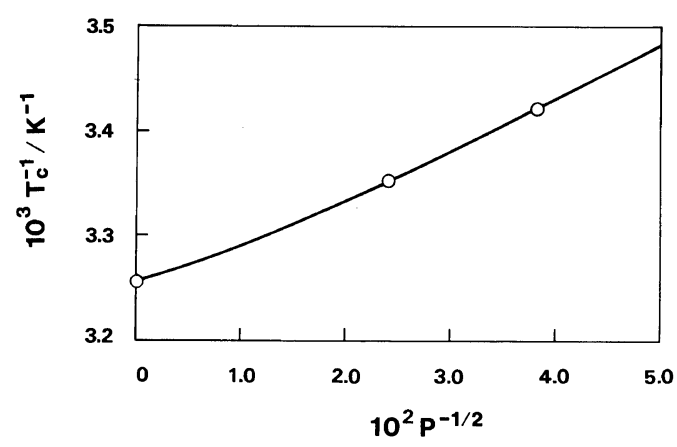

Figure 11. Reciprocal critical temperature $T_{\mathrm{c}}^{-1}$ plotted against $P^{-1 / 2}$ for PIP in dioxane. The solid line shows the theoretical prediction.

and $P^{-1 / 2}$ (subject to the condition $P^{1 / 2} \gg 1$ ) when $\chi$ is constant. It is rather surprising that this relation is essentially obeyed by the systems, treated in the present and previous studies, where $\chi$ depends significantly on $T$, $\phi$, and $P$.

Figure 10 indicates $\phi_{\mathrm{c}}$ to vary almost linearly with $P^{-1 / 3}$. The same $P$ dependence of $\phi_{\mathrm{c}}$ was also experimentally found in our previous work $^{7}$ on the system $\mathrm{PS}+\mathrm{CH}$. It distinctly differs from the linear dependence of $\phi_{\mathrm{c}}$ on $P^{-1 / 2}$ predicted for binary systems with concentration-independent $\chi,{ }^{16}$ suggesting that the critical concentration for binary systems be very sensitive to the concentration dependence of $\chi$. 


\section{CONCLUSIONS}

The present study has shown that the parameter $Z$ for the system PIP + dioxane can be described by the same type of $Z$ expression as derived previously for the system $\mathrm{PS}+\mathrm{CH}$. It was found that when extrapolated to the immiscibility region the resulting expression for $\chi$ allows observed binodals, spinodals, and critical points to be predicted accurately. However, since the parameters involved were determined with only two samples of relatively low molecular weight, it remains to see if $\chi$ in its present form can make similar predictions at higher molecular weights.

The results from this work give additional evidence to what is considered convincing at the present stage; i.e.,

(1) There is a cross-over region of $\phi$ in which the Flory-Huggins interaction parameter $\chi$ for a binary polymer solution changes its concentration dependence rather distinctly.

(2) Even at high concentrations, where the polymer molecules overlap with one another, $\chi$ depends significantly on molecular weight. It seems crucial for a quantitative theoretical prediction of phase separation behavior that these two features, among others, be properly incorporated in the formulation of $\chi$.

\section{REFERENCES}

1. M. Kurata, "Thermodynamics of Polymer Solutions," translated from the Japanese by $\mathrm{H}$. Fujita, Harwood Academic, N. Y., 1982.

2. R. Koningsveld, Thesis, Leiden University, 1967.

3. R. Koningsveld, L. A. Kleintjens, and A. R. Shults, J. Polym. Sci., A-2, 8, 1261 (1970).

4. R. Koningsveld and L. A. Kleintjens, Macromolecules, 4, 637 (1971).

5. R. Koningsveld, W. H. Stockmayer, J. W. Kennedy, and L. A. Kleintjens, Macromolecules, 7, 73 (1974).

6. H. Fujita and A. Teramoto, Proceedings of MMI Symposium on Polymer Compatibility and Imcompatibility, Harwood Academic, N.Y., 1982, p. 125.

7. Y. Einaga, S. Ohashi, Z. Tong, and H. Fujita, Macromolecules, 17, 527 (1984).

8. Y. Einaga, Z. Tong, and H. Fujita, Macromolecules, to be published.

9. Th. G. Scholte, Eur. Polym. J., 6, 1063 (1970).

10. Th. G. Scholte, J. Polym. Sci., A-2, 9, 1553 (1971).

11. L. A. Wood and G. M. Martin, J. Natl. Bur. Stand. Sect. A, 68, 259 (1964).

12. M. Tsuyumoto, Y. Einaga, and H. Fujita, Polym. J., 16, 229 (1984).

13. Z. Tong and Y. Einaga, Polym. J., 16, 641 (1984).

14. Z. Tong, S. Ohashi, Y. Einaga, and H. Fujita, Polym. J., 15, 835 (1983).

15. E. Nies, R. Koningsveld, and L. A. Kleintjens, IUPAC Symposium, Bucharest, 1983.

16. P. J. Flory, "Principles of Polymer Chemistry," Cornell Univ. Press, N.Y., 1953. 